\title{
Iterative Multiuser Detection for Convolutionally Coded Asynchronous DS-CDMA *
}

\author{
Matthew C. Valenti and Brian D. Woerner \\ Mobile and Portable Radio Research Group \\ Virginia Polytechnic Institute and State University \\ Blacksburg, VA 24061-0350, U.S.A. \\ email: valenti@vt.edu, phone: 540-231-2925, fax: 540-231-2968
}

\begin{abstract}
A soft-input, soft-output multiuser detection algorithm based on the Log-MAP algorithm is presented for asynchronous multiple access networks. The algorithm is applied to a convolutionally coded Direct Sequence Code Division Multiple Access (DS-CDMA) system. The proposed receiver architecture consists of a Log-MAP multiuser detector followed by a Log-MAP channel decoder. Information is fed back from the channel decoder to the multiuser detector, and processing proceeds in an iterative fashion analogous to the decoding of turbo codes. Simulation results show that the performance of a heavily loaded power controlled DS-CDMA system approaches the single-user bound for both Additive White Gaussian Noise (AWGN) and fullyinterleaved Rayleigh flat-fading channels.
\end{abstract}

\section{INTRODUCTION}

The conventional receiver for Direct-Sequence Code Division Multiple Access (DS-CDMA) consists of a bank of matched filters, each matched to a particular user's signature waveform. In such a receiver, multiuser interference (MUI) is considered to be noise and is not exploited by the receiver. Performance can be greatly improved by using multiuser detection (MUD), a method of jointly detecting all the users in the system. Multiuser detection algorithms treat MUI as a form of time-varying intersymbol interference (ISI), which is equalized using a variety of techniques. The optimal multiuser detector for asynchronous DS-CDMA is analogous to the Maximum Likelihood Sequence Estimator (MLSE) for ISI, and is implemented using the Viterbi algorithm [1]. Under a wide range of conditions, multiuser detection allows uncoded asynchronous DS-CDMA systems to achieve performance comparable to that of the single-user system.

A major obstacle preventing the use of optimal multiuser detection in coded CDMA systems is the interface between

*This work has been supported by the Bradley Fellowship, the MPRG Industrial Affiliates Foundation, and the Office of Naval Research. the MUD and the channel decoder. If the MUD is implemented with the Viterbi algorithm, then the output of the MUD consists of "hard" bit decisions. However, it is preferable to pass unquantized values, or "soft" bit decisions, into the channel decoder. The decoder for convolutional codes, which is also implemented with the Viterbi algorithm, suffers a $2.5 \mathrm{~dB}$ loss in coding gain when hard bit decisions are used as inputs instead of soft decisions [2]. In section III, we present a soft-output MUD algorithm that greatly improves the interface between MUD and channel decoder.

Even if soft-output multiuser detection is used, system performance is not optimal in the sense that the MUD cannot benefit from information derived by the channel decoder. In [3], the problem of joint multiuser detection and convolutional decoding is approached by defining a supertrellis over the cross product of the channel and encoder states, and then executing a single instance of the Viterbi algorithm over the entire supertrellis. While this offers performance close to the coded single-user bound, it has complexity $O\left(2^{K W}\right)$ ( $W$ is the constraint length of the convolutional code), does not lend itself to systems that incorporate interleaving, and cannot be easily mapped onto parallel processing architectures. An alternative approach is to partition the problem into two parts, multiuser detection and channel decoding. The MUD and channel decoder are each implemented with a soft-input, soft-output (SISO) algorithm and operate in an iterative feedback mode whereby the information derived by the channel decoder is fed back to the MUD. This approach is analogous to the method of decoding turbo codes [4] and has been applied to joint MUD and channel decoding for synchronous DS-CDMA in [5] and for asynchronous Time Division Multiple Access (TDMA) in [6].

In this paper, a method of performing combined multiuser detection and channel decoding for asynchronous DS-CDMA systems is proposed. This method uses SISO multiuser detection and channel decoding implemented by the log-MAP algorithm of [7] and operates in an iterative feedback, or "turbo processing" mode. In section II, the system model is presented. In section III, the receiver architecture and 
log-MAP MUD algorithm are proposed. The receiver architecture is similar to the system proposed in [5]. However, because we consider systems that are bit and chip asynchronous, the structure of the MUD is significantly different. The synchronous system of [5] allowed a one-shot approach to MUD, while the asynchronous system considered in this paper requires sequence detection. Simulation results for a heavily loaded convolutionally coded CDMA system are given in section IV for both Additive White Gaussian Noise (AWGN) and fully-interleaved Rayleigh flat-fading channels. Finally conclusions are given in section $\mathrm{V}$.

\section{SYSTEM MODEL}

Consider a CDMA network consisting of $K$ asynchronous BPSK transmitters and a single receiver. At transmitter $k$, a bit sequence, $\mathbf{d}_{k}=\left\{d_{k}[j]\right\}$ for $1 \leq j \leq L / n$, the last $m_{c}$ of which are zeros, is passed through a rate $R=1 / n$ convolutional encoder with constraint length $W=m_{c}+1$. The coded symbols are interleaved, BPSK modulated, and sent over a (possibly flat-fading) multiple access channel. The signal at the receiver is the noisy sum of $K$ delayed and attenuated signals generated by each of the $K$ transmitters

$$
r(t)=\sum_{k=1}^{K} s_{k}(t)+n(t),
$$

where $n(t)$ is a complex Additive White Gaussian Noise (AWGN) process with power spectral density $N_{o} / 2$ and $s_{k}(t)$ is the signal at the receiver due to user $k$

$$
s_{k}(t)=\sum_{i=1}^{L} \sqrt{P_{k}[i]} b_{k}[i] a_{k}\left(t-i T-\tau_{k}\right) e^{j \phi_{k}} .
$$

$L$ is the block size, $P_{k}[i]$ is the instantaneous power of user $k$ at the receiver during the $i^{t h}$ signaling interval, $b_{k}[i] \in\{-1,1\}$ is the $i^{\text {th }}$ coded and interleaved symbol of user $k, \tau_{k}$ is the delay of user $k$ at the receiver assumed to be uniformly distributed over one symbol period $T$, and $\phi_{k}$ is the carrier phase of user $k$ at the receiver assumed to be uniformly distributed over the interval $(0,2 \pi)$. The signal $a_{k}(t)$ is the signature waveform for user $k$ and is composed of $N$ chips, where $N$ is the processing gain of the CDMA system. For ease of exposition, it is assumed that the signature sequences repeat every symbol period. However, the algorithm presented in this paper can be easily extended to incorporate long codes (i.e. codes that do not repeat every symbol period). It is assumed that the indices of the $K$ users are assigned such that the relative delays $\tau_{k}$ are in ascending order, i.e. $\tau_{1} \leq \tau_{2} \leq \ldots \leq \tau_{K}$. For AWGN channels, the power $P_{k}[i]$ is constant for all $i$. For fully-interleaved Rayleigh flat-fading channels, each amplitude $\sqrt{P_{k}[i]}$ is an independent realization of a Rayleigh random variable. Fading is assumed to be slow, and thus the fading amplitude is constant for the entire symbol interval.

The received signal is passed through a bank of $K$ matched filters with output

$$
y_{k}[i]=\int_{-\infty}^{\infty} \Re\left\{r(t) a_{k}\left(t-i T-\tau_{k}\right) e^{-j \phi_{k}}\right\} d t,
$$

where $y_{k}[i]$ denotes the matched filter output corresponding to the $i^{t h}$ code symbol of user $k$, and $\Re\{\cdot\}$ denotes the real part. The vector $\mathbf{y}$ of matched filter outputs constitutes a set of sufficient statistics and is created in "round-robin" fashion

$$
\mathbf{y}=\left[y_{1}[1], \ldots, y_{K}[1], \ldots, y_{1}[L], \ldots, y_{K}[L]\right]^{T} .
$$

Similarly, a vector $\mathbf{b}$ is defined by

$$
\mathbf{b}=\left[b_{1}[1], \ldots, b_{K}[1], \ldots, b_{1}[L], \ldots, b_{K}[L]\right]^{T} .
$$

The fading amplitudes are also combined into a vector

$$
\mathbf{c}=\left[\sqrt{P_{1}[1]}, \ldots, \sqrt{P_{K}[1]}, \ldots, \sqrt{P_{1}[L]}, \ldots, \sqrt{P_{K}[L]}\right]^{T} .
$$

Finally, a $(K-1)$ by $K$ matrix of signal cross correlations is defined by

$$
\begin{aligned}
G_{i, j}= & \frac{1}{T} \cos \left(\phi_{i+j}-\phi_{j}\right) \\
& \cdot \int_{-\infty}^{\infty} a_{i+j}\left(t-\tau_{i+j}\right) a_{j}\left(t-\tau_{j}-T\right) d t,
\end{aligned}
$$

if $i+j \leq K$, and

$$
\begin{aligned}
G_{i, j}= & \frac{1}{T} \cos \left(\phi_{i+j-K}-\phi_{j}\right) \\
& \cdot \int_{-\infty}^{\infty} a_{i+j-K}\left(t-\tau_{i+j-K}\right) a_{j}\left(t-\tau_{j}\right) d t,
\end{aligned}
$$

if $i+j>K$. The entries of the $j^{t h}$ column of $G$ are the correlations (at the receiver) of the $j^{\text {th }}$ user with the $K-1$ preceeding users.

\section{ITERATIVE MUD FOR ASYNCHRONOUS DS-CDMA}

The proposed turbo processing architecture is shown in Figure 1. The matched filter outputs $\mathbf{y}$ are fed into a SISO multiuser detector, which produces a vector $\Psi^{(q)}$ of loglikelihood ratios (LLRs). The LLRs are then deinterleaved and distributed to a bank of $K$ SISO channel decoders, which produces the vector $\Lambda^{\left(q^{\prime}\right)}$ containing the LLRs of the code bits. These LLRs are reinterleaved and sent back to the MUD to be used as a priori information for iteration $q+1$. The hard system output after the $q^{t h}$ iteration is the sign of the LLRs $\boldsymbol{\Omega}^{\left(q^{\prime}\right)}$ of the data bits, and is denoted $\hat{\mathbf{d}}^{(q)}$.

\section{A. Multiuser Detection}

The optimal multiuser detector for iteration $q$ selects the vector of estimated transmitted bits according to [1]

$$
\hat{\mathbf{b}}^{(q)}=\arg \left\{\max _{\mathbf{b}} \sum_{i=1}^{K L} \lambda_{i}(\mathbf{b})\right\},
$$




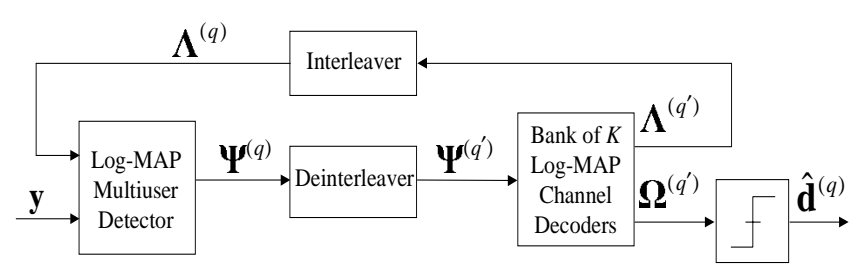

Figure 1: Proposed reception technique.

where

$$
\begin{aligned}
\lambda_{i}(\mathbf{b})= & \frac{n}{E_{b} / N_{o}} \ln P\left[b_{i} \mid \boldsymbol{\Lambda}^{(q-1)}\right] \\
& +b_{i} c_{i}\left[2 y_{i}-b_{i} c_{i}-2 \sum_{d=1}^{K-1} b_{i-d} c_{i-d} G_{K-d, \kappa(i)}\right],
\end{aligned}
$$

with $E_{b}$ the average energy per data bit and $b_{i}=0$ for $i \leq 0 . \kappa(i)=K$ if $K$ divides $i$, otherwise $\kappa(i)$ is $i$ modulo $K$. $P\left[b_{i} \mid \Lambda^{(q-1)}\right]$ is related to the soft output of the channel decoder during the $(q-1)^{t h}$ iteration by

$$
P\left[b_{i} \mid \Lambda^{(q-1)}\right]= \begin{cases}\frac{\exp \Lambda_{i}^{(q-1)}}{1+\exp \Lambda_{i}^{(q-1)}} & \text { if } b_{i}=+1 \\ \frac{1}{1+\exp \Lambda_{i}^{(q-1)}} & \text { if } b_{i}=-1\end{cases}
$$

During initialization, $\boldsymbol{\Lambda}^{(0)}$ is set to be all zeros. The estimate $\hat{\mathbf{b}}_{m}$ can be recursively computed using the Viterbi algorithm, using the branch metric given by (10).

\section{B. $\log -M A P M U D$}

Use of the standard Viterbi algorithm with metric given by (10) results in hard bit decisions, which are not suitable for turbo processing. Instead, soft outputs from the MUD in the form

$$
\Psi_{i}^{(q)}=\ln \frac{P\left[b_{i}=+1 \mid \mathbf{y}, \boldsymbol{\Lambda}^{(q-1)}\right]}{P\left[b_{i}=-1 \mid \mathbf{y}, \boldsymbol{\Lambda}^{(q-1)}\right]}
$$

are desired. This LLR can be calculated using the Maximum A Posteriori (MAP) algorithm of [8]. Although the MAP algorithm offers optimal estimates of (12), it suffers from a high computational complexity and numerical instability. The log-MAP algorithm of [7] performs the MAP algorithm in the log domain and thus has reduced complexity and is more numerically stable. In addition, there are several suboptimal approaches to calculating (12) that go beyond the scope of this paper. Due to its numerical stability and relatively low complexity, we only consider the $\log$-MAP algorithm for the remainder of this paper. The log-MAP algorithm was originally proposed to decode turbo codes, and therefore must be appropriately modified for use as a soft-output multiuser detector. We follow the treatment of the log-MAP algorithm in [9], which describes the $\log$-MAP algorithm as an instance of two generalized Viterbi algorithms, one operating in a forward recursion the other working in a backward recursion.

For the forward recursion, a trellis $\alpha_{i}(m)$ is initialized according to

$$
\alpha_{0}(m)= \begin{cases}0, & m=0 \\ -\infty . & m \neq 0\end{cases}
$$

where the node of the trellis is identified by the time index $i \in[0, \ldots, K L]$ and state index $m \in\left[0, \ldots, 2^{K-1}\right]$. The remainder of the trellis is populated by the generalized Viterbi algorithm defined by the recursion relation

$$
\alpha_{i}(s)=\max _{s^{\prime}} *\left[\alpha_{i-1}\left(s^{\prime}\right)+\gamma_{i-1}\left(s^{\prime}, s\right)\right] .
$$

In the above, the operator max $*$ is defined by the Jacobian logarithm

$$
\begin{aligned}
\max *(x, y) & =\ln \left(e^{x}+e^{y}\right) \\
& =\max (x, y)+\ln \left(1+e^{-|x-y|}\right),
\end{aligned}
$$

and by the recursion relationship

$$
\max *(x, y, z)=\max *(x, \max *(y, z))
$$

To compute (16) a correction factor is added to the maximum of the two arguments. Note that the correction factor depends only on the difference of the arguments, and is negligible except when $x$ and $y$ have similar values. The correction factor can be stored in a one-dimensional lookup table, and acceptable performance can be achieved with just eight table entries. If no correction factor is used, then the algorithm is the max-log-MAP algorithm [7].

The factor $\gamma_{i-1}\left(s^{\prime}, s\right)$ in (14) is the branch metric that connects the node at time index $i-1$ and state $s^{\prime}$ to the node at time index $i$ and state $s . \gamma$ and $\lambda$ are related by

$$
\gamma_{i}\left(s^{\prime}, s\right)=\lambda_{i}(\mathbf{b}), \mathbf{b}: s^{\prime} \rightarrow s .
$$

For the backward recursion, a trellis $\beta_{i}(m)$ is initialized according to

$$
\beta_{K L}(m)= \begin{cases}0, & m=0 \\ -\infty . & m \neq 0,\end{cases}
$$

if the MUI trellis is terminated, otherwise $\beta_{i}(m)$ is initialized such that $\beta_{K L}(m)=0$ for all states $m$. Trellis termination occurs if each user sends a final zero or "guard symbol" at the end of each frame, i.e. if $b_{k}[L]=-1, \forall k$. The remainder of the trellis is populated by a generalized Viterbi algorithm running according to the backward recursion:

$$
\beta_{i}(s)=\max _{s^{\prime}} *\left[\beta_{i+1}\left(s^{\prime}\right)+\gamma_{i}\left(s, s^{\prime}\right)\right] .
$$

Once the forward and backward recursions have been completed, the LLR is found by

$$
\begin{aligned}
\Psi_{i}^{(q)}= & \max _{\left(s^{\prime}, s\right) \in S_{1}} *\left[\alpha_{i-1}\left(s^{\prime}\right)+\gamma_{i-1}\left(s^{\prime}, s\right)+\beta_{i}(s)\right] \\
& -\max _{\left(s^{\prime}, s\right) \in S_{0}} *\left[\alpha_{i-1}\left(s^{\prime}\right)+\gamma_{i-1}\left(s^{\prime}, s\right)+\beta_{i}(s)\right],
\end{aligned}
$$


where $S_{1}$ is the set of all state transitions $s^{\prime} \rightarrow s$ associated with $b_{i}=+1$ and $S_{0}$ is the set of all $s^{\prime} \rightarrow s$ asociated with $b_{i}=-1$. The soft-output of the MUD is deinterleaved, and the resulting sequence $\boldsymbol{\Psi}^{\left(q^{\prime}\right)}$ is passed to the channel decoder.

\section{Channel Decoding}

The serial output, $\Psi^{\left(q^{\prime}\right)}=\left\{\Psi_{i}^{\left(q^{\prime}\right)}\right\}$ for $1 \leq i \leq K L$, of the deinterleaver is split into $K$ parallel sequences $\left\{\boldsymbol{\Psi}_{k}^{\left(q^{\prime}\right)}\right\}$, where $\boldsymbol{\Psi}_{k}^{\left(q^{\prime}\right)}=\left\{\Psi_{(i-1) K+k}^{\left(q^{\prime}\right)}\right\}$ for $1 \leq i \leq L$. The information $\boldsymbol{\Psi}_{k}^{\left(q^{\prime}\right)}$ for user $k$ is fed into a soft-output channel decoder, which produces two output sequences. The first output is the log-likelihood ratio of the $k^{t h}$ user's channel encoder output

$$
\Lambda_{k}^{\left(q^{\prime}\right)}[i]=\ln \frac{P\left[b_{k}[i]=+1 \mid \Psi_{k}^{\left(q^{\prime}\right)}\right]}{P\left[b_{k}[i]=-1 \mid \Psi_{k}^{\left(q^{\prime}\right)}\right]} .
$$

The outputs of the $K$ channel decoders are combined into a vector according to

$$
\Lambda^{\left(q^{\prime}\right)}=\left[\Lambda_{1}^{\left(q^{\prime}\right)}[1], \ldots, \Lambda_{K}^{\left(q^{\prime}\right)}[1], \ldots, \Lambda_{1}^{\left(q^{\prime}\right)}[L], \ldots, \Lambda_{K}^{\left(q^{\prime}\right)}[L]\right]^{T} .
$$

This vector is fed back to the MUD and is used as a priori information during the $(q+1)^{t h}$ iteration of turbo processing.

The second output of the channel decoder for user $k$ is the log-likelihood ratio of the channel encoder input, and is given by

$$
\Omega_{k}^{\left(q^{\prime}\right)}[j]=\ln \frac{P\left[d_{k}[j]=+1 \mid \Psi_{k}^{\left(q^{\prime}\right)}\right]}{P\left[d_{k}[j]=-1 \mid \Psi_{k}^{\left(q^{\prime}\right)}\right]} .
$$

Several soft-output algorithms can be used to implement the channel decoder. For the purposes of this study, we consider the use of the log-MAP algorithm to perform channel decoding.

\section{SIMULATION RESULTS}

The performance of the proposed reception technique was simulated for a convolutionally coded DS-CDMA system. The convolutional code chosen has rate $R=1 / 2$, constraint length $W=3$, and octal generator $(7,5)$. The CDMA system has processing gain $N=7$ and the signature sequences were randomly assigned. The number of users of the CDMA system is $K=5$. The frame size is 264 data bits, which includes two tail bits to terminate the trellis of the encoder. The block of $L=528$ code bits is passed through a 24 by 22 block interleaver prior to transmission. A guard bit is added to the end of each user's frame to terminate the trellis of the MUD. It is assumed that the users are power controlled in the sense that the average power at the receiver is the same

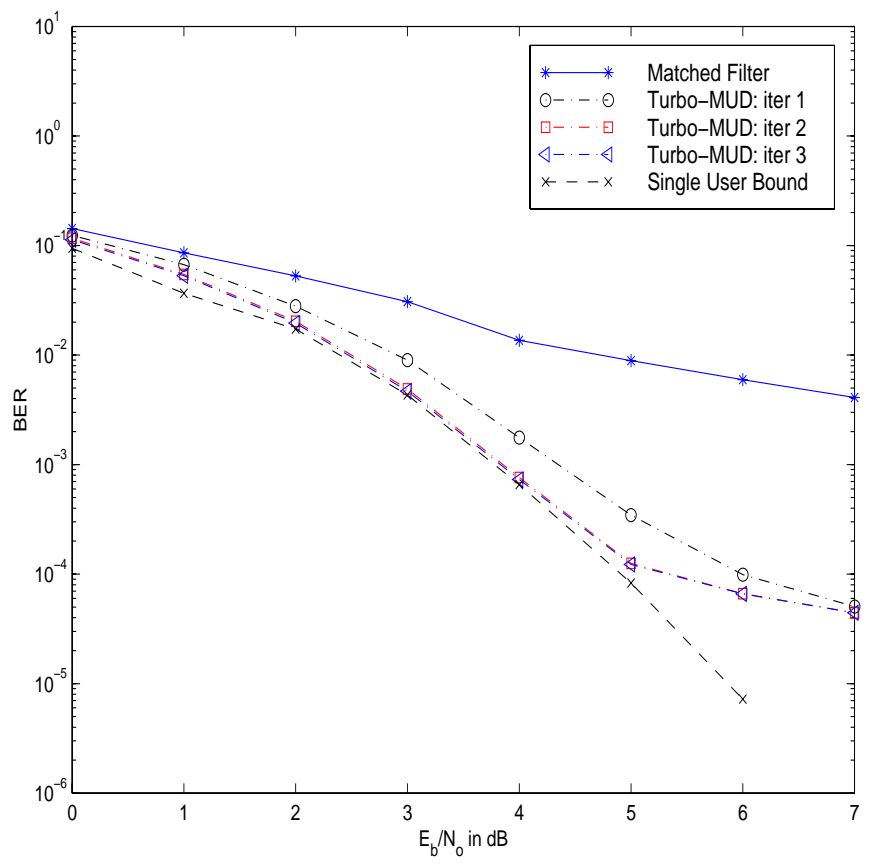

Figure 2: Bit error performance of a $K=5$ user, $N=$ 7 processing gain DS-CDMA system in an AWGN channel with rate $R=1 / 2$, constraint length $W=3$ convolutional coding as parameterized by decoding technique.

for all users. Both the MUD and the channel decoder are implemented with the log-MAP algorithm using a lookup table with eight entries for the correction factor. Both AWGN and fully-interleaved Rayleigh flat-fading channels were considered. For each case, enough trials were run to generate 100 frame errors.

The simulation results for the AWGN channel are shown in Figure 2. The upper curve of this plot shows the performance of the conventional receiver for coded CDMA, which consists of a bank of matched filters followed by the standard Viterbi algorithm for channel decoding. The lower curve of the plot shows the performance of a convolutionally coded single-user system. The performance of the single-user system is limited solely by noise, since there are no interfering users. The three curves in the middle of the plot show the performance of the proposed turbo processing technique. The curve for iteration one shows the performance when a Log-MAP multiuser detector is followed by a Log-MAP channel decoder. The curves for iteration two and three show the performance when the LLR's generated by the logMAP channel decoder are fed back to the MUD as a priori information. Note that there is a significant gain achieved when one iteration of processing is used, and a moderate gain when a second iteration is performed. However, the gain of additional iterations beyond the second is negligible. For bit error rates between $10^{-2}$ and $10^{-4}$ performance after the second iteration is very close to the single user bound. However, as the bit error rate drops below $10^{-4}$ the per- 


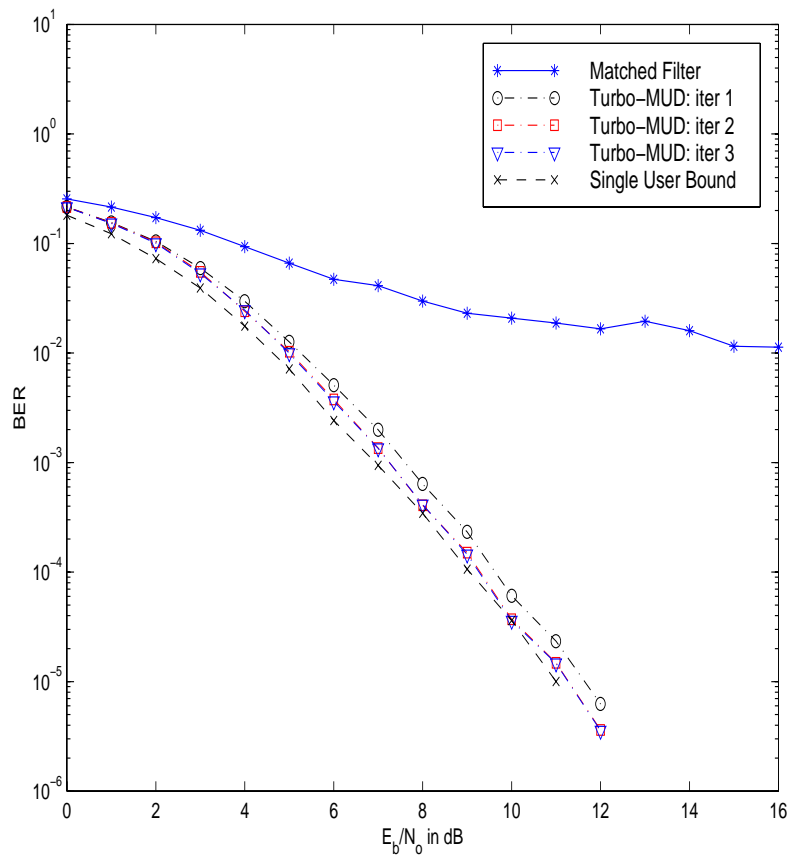

Figure 3: Bit error performance of a $K=5$ user, $N=7$ processing gain DS-CDMA system in a fully-interleaved Rayleigh flat-fading channel with rate $R=1 / 2$, constraint length $W=3$ convolutional coding as parameterized by decoding technique.

formance becomes interference limited and begins to move away from the single user bound. To improve performance in this region, either a stronger convolutional code must be used or the number of users must be decreased.

The simulation results for the fully-interleaved Rayleigh flatfading channel are given in Figure 3. The performance of the coded matched filter receiver reaches a floor of approximately $10^{-2}$ due to multiuser interference and fading. The performance of the turbo receiver is considerably better, and after two iterations is within $0.5 \mathrm{~dB}$ of the single user bound for Rayleigh fading and the same convolutional code. It is interesting to note that unlike for the AWGN case, performance remains close to the single user bound even as the bit error rate drops below $10^{-4}$. This behavior can be accounted for by the fact that with flat-fading, the instantaneous received powers of the users are generally different. Unlike the AWGN case, where the received powers are the same for all users at all times, the Rayleigh fading case gives the multiuser detector one more signal parameter, instantaneous received power, that it can use to differentiate the users. This does not imply that the performance over flatfading channels will be better than over AWGN channels, only that the gain from using multiuser detection for fading channels is greater for fading channels than it is for AWGN channels with power control.

\section{CONCLUSION}

In this paper, a strategy for combining multiuser detection with channel decoding for asynchronous DS-CDMA systems was proposed. The method is based on the principle of turbo processing, whereby the problem of joint multiuser detection and channel decoding is separated into soft-intput, softoutput MUD and channel decoding processes that operate in an iterative feedback manner. Simulation results were shown for a heavily loaded CDMA system in AWGN and Rayleigh flat-fading. For both channels, performance after the second iteration was close to the single user bound. As the bit error rate fell below $10^{-4}$ a floor was reached for the AWGN case, but this floor was not observed for the Rayleigh flat fading channel. The complexity of the proposed strategy is $O\left(Q\left(2^{K}+2^{W}\right)\right)$, where $W$ is the code constraint length, $K$ the number of users, and $Q$ the number of iterations. This is preferable to the complexity of the supertrellis-based method of [3], which had complexity $O\left(2^{K W}\right)$. While this work has allowed the complexity of the joint multiuser detection, channel decoding problem to be greatly reduced, future work should concentrate on reducing the complexity further.

\section{REFERENCES}

[1] S. Verdu, "Minimum Probability of Error for Asynchronous Gaussian Multiple Access Channels," IEEE Trans. Info. Theory, vol. 32, no. 1, pp. 85-96, Jan. 1986.

[2] J. Proakis, Digital Communications, McGraw Hill, 1995.

[3] T. R. Giallorenzi and S. G. Wilson, "Multiuser ML sequence estimator for convolutionally coded asynchronous DS-CDMA systems," IEEE Trans. Commun., pp. 997-1008, Aug. 1996.

[4] C. Berrou, A. Glavieux, and P. Thitimasjshima, "Near Shannon limit error-correcting coding and decoding: Turbo-codes(1)," in Proc., IEEE Int. Conf. on Commun. (Geneva Switzerland, 1993), pp. 1064-1070.

[5] M.C. Reed, P.D. Alexander, J.A. Asenstorfer, and C.B. Schlegel, "Iterative multiuser detection for CDMA with FEC," in Proc., Int. Symp. on Turbo Codes 83 Related Topics, (Brest France), pp. 162-165.

[6] M.C. Valenti and B.D. Woerner, "Combined multiuser reception and channel decoding for TDMA cellular systems," in Proc., IEEE Vehicular Technology Conf. (Ottawa Canada, 1998), pp. 1915-1919.

[7] P. Robertson, P. Hoeher, and E. Villebrun, "Optimal and sub-optimal maximum a posteriori algorithms suitable for turbo decoding," European Trans. on Telecommun., vol. 8, pp. 119-125, Mar./Apr. 1997.

[8] L.R. Bahl, J. Cocke, F. Jeinek, and J. Raviv, "Optimal decoding of linear codes for minimizing symbol error rate," IEEE Trans. Inform. Theory, vol. 20, pp. 284287, Mar. 1974.

[9] A. J. Viterbi, "An intuitive justification and a simplified implementation of the MAP decoder for convolutional codes," IEEE J. Select. Areas Commun., vol. 16, no. 2, pp. 260-264, Feb. 1998. 